\title{
Compactness and hypercyclicity of co-analytic Toeplitz operators on de Branges-Rovnyak spaces
}

https://doi.org/10.1515/conop-2020-0004

Received September 19, 2019; accepted January 27, 2020

\begin{abstract}
We study the compactness and the hypercyclicity of Toeplitz operators $T_{\bar{\varphi}, b}$ with co-analytic and bounded symbols on de Branges-Rovnyak spaces $\mathcal{H}(b)$. For the compactness of $T_{\bar{\varphi}, b}$, we will see that the result depends on the boundary spectrum of $b$. We will prove that there are non trivial compact operators of the form $T_{\bar{\varphi}, b}$, with $\varphi \in H^{\infty} \cap C(\mathbb{T})$, if and only if $m(\sigma(b) \cap \mathbb{T})=0$. We will also show that, when $b$ is non-extreme, then $T_{\bar{\varphi}, b}$ is hypercyclic if and only if $\varphi$ is non-constant and $\varphi(\mathbb{D}) \cap \mathbb{T} \neq \emptyset$.
\end{abstract}

Keywords: Toeplitz operators, de Branges-Rovnyak spaces, compactness, hypercyclicity

MSC: 30J05,30H10,46E22,47A16

\section{Introduction}

We shall mostly be discussing co-analytic Toeplitz operators $T_{\bar{\varphi}}$ with symbol $\bar{\varphi}$ where $\varphi \in H^{\infty}$, that are naturally defined on the de Branges-Rovnyak space into itself. These operators have been introduced by LottoSarason in [13, Lemma 2.6], see also [14, Section II.7]. Some special cases have long ago appeared in literature for $\varphi \in L^{\infty}(\mathbb{T})$, most notably as standard Toeplitz operators $T_{\varphi}: H^{2} \rightarrow H^{2}$ studied by A. Brown and P. Halmos in the paper [5] and as the adjoints of truncated Toeplitz operators $A_{\varphi}^{\Theta}$ on model spaces $K_{\Theta}$ introduced by Sarason in [15]. We will consider Toeplitz operators with different domains and different ranges. To avoid confusion, we adopt different notations. We will denote by $T_{\bar{\varphi}}$ the Toeplitz operator defined from $H^{2}$ into itself, by $T_{\bar{\varphi}, b}$ the Toeplitz operator defined from $\mathcal{H}(b)$ into itself, and by $\mathbf{T}_{\bar{\varphi}, b}$ the Toeplitz operator defined from $\mathcal{H}(b)$ into $H^{2}$.

It turns out that de Branges-Rovnyak spaces, which are a family of subspaces $\mathcal{H}(b)$ of the Hardy space $H^{2}$, parametrized by elements $b$ of the closed unit ball of $H^{\infty}$ are invariant under $T_{\bar{\varphi}}$, where $\varphi \in H^{\infty}$. We shall give the precise definition in section 2. In general $\mathcal{H}(b)$ is not closed in $H^{2}$, but it carries its own norm $\|\cdot\|_{\mathcal{H}(b)}$ making it a Hilbert space. The spaces $\mathcal{H}(b)$ were introduced by de Branges and Rovnyak in the appendix of [6] and further studied in their book [7].

The general theory of $\mathcal{H}(b)$-spaces generally splits into two cases, according to whether $b$ is an extreme point or a non-extreme point of the unit ball of $H^{\infty}$. The dichotomy $b$ extreme/non-extreme will also often appear in this paper. The general idea is that the extreme case has many features that are not far from the case of $b=\Theta$ inner (the classical model space $K_{\Theta}$ ), while the non-extreme case has several properties that are similar to the case where $b=0$ (the Hardy space $H^{2}$ ).

This paper treats two properties related to the restricted Toeplitz operators $T_{\bar{\varphi}, b}$ when $\varphi \in H^{\infty}$. One of these properties is based on the particular operator $X_{b}=T_{\bar{z}, b}$ that plays a central role in the theory and

^Corresponding Author: Rim Alhajj: Laboratoire Paul Painlevé, Université Lille 1, 59655 Villeneuve d’Ascq Cédex, France, E-mail: rim.alhajj@univ-lille.fr 
particularly in the model developed by de Branges and Rovnyak. Indeed, it serves as a model for a large class of contractions (see [8, Theorem 26.16]).

The first one concerns the compactness of the Toeplitz operator $T_{\bar{\varphi}, b}$, More precisely, given an element $b$ of the closed unit ball of $H^{\infty}$, that is extreme or non-extreme, we are interested in finding a necessary and sufficient condition for the operator $T_{\bar{\varphi}, b}$ to be compact. Let us mention that Brown and Halmos [5] have shown that there is no compact Toeplitz operators $T_{\varphi}: H^{2} \rightarrow H^{2}$ with symbol $\varphi \in L^{\infty}(\mathbb{T})$ (except the trivial case where $\varphi=0$ ). It is not surprising that in the case where $b$ is non-extreme, we reach a similar result for the restricted Toeplitz operator $T_{\bar{\varphi}, b}$ with $\varphi \in H^{\infty}$.

On the other hand, Ahern and Clark [1] studied the compactness of truncated Toeplitz operators $A_{\varphi}^{\Theta}$, with continuous symbol $\varphi$ on $\mathbb{T}$ and they got a necessary and sufficient condition based on the image of the spectrum of $\Theta$ intersected with the unit circle. Recently, Garcia, Ross and Wogen [9] recovered this result with another proof. Using their method, we generalize the result of Ahern and Clark to the operator $\mathbf{T}_{\bar{\varphi}, b}$ with $\varphi \in C(\mathbb{T})$, and from this generalization we prove that there are non trivial compact operators of the form $T_{\bar{\varphi}, b}$, with $\varphi \in H^{\infty} \cap C(\mathbb{T})$, if and only if $m(\sigma(b) \cap \mathbb{T})=0$.

Our second problem is related to the hypercyclicity of the restricted Toeplitz operator $T_{\bar{\varphi}, b}$. Godefroy and Shapiro, using their Criterion [11], proved that if $\varphi \in H^{\infty}$, then $T_{\bar{\varphi}}$ is hypercyclic if and only if $\varphi$ is non constant and $\varphi(\mathbb{D}) \cap \mathbb{T} \neq \emptyset$. On the other hand, it is obvious that there are no hypercyclic Toeplitz operators with analytic symbols. For general symbols $\varphi \in L^{\infty}(\mathbb{T})$, few results are known; see a recent paper of A.Baranov and A. Lishanskii [2] and a paper of Shkarin [16] who studied the case where $\varphi(z)=a \bar{z}+b+c z$. We succeed to extend the result of Godefroy and Shapiro in our context of $\mathcal{H}(b)$ spaces, when $b$ is non extreme. Whereas, when $b$ is extreme, we give a necessary condition for the operator $T_{\bar{\varphi}, b}$ to be non-hypercyclic. This condition is based on the point spectrum of the operator. It also turns out that this necessary condition is not sufficient.

The structure of the paper is the following. After a preliminary section with generalities about de Branges-Rovnyak spaces, Toeplitz operators and definitions of hypercyclic and frequently hypercyclic operators, we discuss compactness properties of the restricted Toeplitz operators $T_{\bar{\varphi}, b}$ in Section 3. The last section is dedicated to the hypercyclicity of $T_{\bar{\varphi}, b}$.

\section{Preliminaries}

\subsection{Toeplitz operators and de Branges-Rovnyak spaces}

We first recall some basic facts on Toeplitz operators on the Hardy space $H^{2}$ of the open unit disc $\mathbb{D}=\{z \in$ $\mathbb{C}:|z|<1\}$.

Given $\varphi \in L^{\infty}(\mathbb{T})=L^{\infty}(\mathbb{T}, m)$ where $\mathbb{T}=\partial \mathbb{D}$ and $m$ is the normalized Lebesgue measure on $\mathbb{T}$, the corresponding Toeplitz operator $T_{\varphi}: H^{2} \rightarrow H^{2}$ is defined by

$$
T_{\varphi} f:=P_{+}(\varphi f) \quad\left(f \in H^{2}\right),
$$

where $P_{+}: L^{2}(\mathbb{T}) \rightarrow H^{2}$ denotes the othogonal projection of $L^{2}(\mathbb{T})=L^{2}(\mathbb{T}, m)$ onto $H^{2}$. Clearly $T_{\varphi}$ is a bounded operator on $H^{2}$ with $\left\|T_{\varphi}\right\|=\|\varphi\|_{L^{\infty}(\mathbb{T})}$, moreover it is compact if and only if $\varphi=0$ (Brown-Halmos, [5]). If $\varphi \in$ $H^{\infty}$ the algebra of the analytic and bounded functions on $\mathbb{D}$, then $T_{\varphi}$ is simply the operator of multiplication by $\varphi$ and its adjoint is $T_{\bar{\varphi}}$. Consequently, if $\varphi, \psi \in H^{\infty}$, then $T_{\bar{\varphi}} T_{\bar{\psi}}=T_{\bar{\varphi} \bar{\psi}}=T_{\bar{\psi}} T_{\bar{\varphi}}$.

Moreover, if $\varphi \in H^{\infty}$ we have

$$
T_{\bar{\varphi}} k_{\lambda}=\overline{\varphi(\lambda)} k_{\lambda},
$$

where $k_{\lambda}(z)=(1-\bar{\lambda} z)^{-1}$ is the reproducing kernel of $H^{2}$ (see [8, Section 12.4]). 
If $\varphi \in L^{\infty}(\mathbb{T})$ satisfies $\|\varphi\|_{\infty} \leq 1$, then the corresponding Toeplitz operator $T_{\varphi}$ is a contraction on the Hilbert space $H^{2}$. The associated de Branges-Rovnyak space $\mathcal{H}\left(T_{\varphi}\right)$ is defined by

$$
\mathcal{H}\left(T_{\varphi}\right)=\left(I-T_{\varphi} T_{\bar{\varphi}}\right)^{1 / 2} H^{2} .
$$

For simplicity, we denote the complementary space $\mathcal{H}\left(T_{\varphi}\right)$ by $\mathcal{H}(\varphi)$ (see [8, Section 17.3]). Therefore, the definition of an $\mathcal{H}(\varphi)$-space uses the defect of the contraction $T_{\varphi}$ [8]. Hence, no doubt, the Toeplitz operators are extremely important in this context. Our main concern is when $\varphi$ is a nonconstant analytic function in the closed unit ball of $H^{\infty}$. In this case, by tradition, we use $b$ instead of $\varphi$.

We recall an alternative and equivalent definition based on reproducing kernels. Namely, $\mathcal{H}(b)$ is the Hilbert space of analytic functions on $\mathbb{D}$ whose reproducing kernel is given by

$$
k_{\lambda}^{b}(z)=\frac{1-\overline{b(\lambda)} b(z)}{1-\bar{\lambda} z}, \quad \lambda, z \in \mathbb{D} .
$$

That is,

$$
f(\lambda)=<f, k_{\lambda}^{b}>_{b}, \forall f \in \mathcal{H}(b), \forall \lambda \in \mathbb{D} .
$$

For $b=0$, we see that $k_{\lambda}^{b}$ coincides with $k_{\lambda}$ the reproducing kernels of $H^{2}$, given by $k_{\lambda}(z)=(1-\bar{\lambda} z)^{-1}$, whence $\mathcal{H}(0)=H^{2}$.

More generally when $\|b\|_{\infty}<1, \mathcal{H}(b)$ coincides with the Hardy space $H^{2}$ with an equivalent norm.

For $b=\Theta$, with $\Theta$ an inner function (that is a function in the closed unit ball of $H^{\infty}$ such that $|\Theta(\zeta)|=1$ almost everywhere on $\mathbb{T}=\partial \mathbb{D})$, the space $\mathcal{H}(\Theta)$ is a closed subspace of $H^{2}$, and we have

$$
\mathcal{H}(\Theta)=\left(\Theta H^{2}\right)^{\perp}:=\left\{f \in H^{2}:\langle f, \Theta g\rangle_{2}=0, \forall g \in H^{2}\right\} .
$$

The space $\mathcal{H}(\Theta)$ is also called the model space and is denoted by $K_{\Theta}=\mathcal{H}(\Theta)$. By Beurling's theorem, the spaces $K_{\Theta}$ correspond to the lattice of closed, non trivial, invariant subspaces for the backward shift operator $S^{\star}=T_{\bar{z}}$ on $H^{2}$.

In the general case, the spaces $\mathcal{H}(b)$ are Hilbert spaces that are contained contractively in $H^{2}$. Moreover, it is well-known that there are relations between the inner products of $\mathcal{H}(b)$ and its cousin $\mathcal{H}(\bar{b})$ since these relations are special cases of the Lotto-Sarason theorem [8, Theorem 16.18 and corollary 16.19]. For further reference, we restate this result below.

Theorem 2.1 ([8], Theorem 17.8). Let $f \in H^{2}$. Then $f \in \mathcal{H}(b)$ if and only if $T_{\bar{b}} f \in \mathcal{H}(\bar{b})$ and

$$
<f_{1}, f_{2}>_{b}=<f_{1}, f_{2}>_{2}+<T_{\bar{b}} f_{1}, T_{\bar{b}} f_{2}>_{\bar{b}}, \quad\left(f_{1}, f_{2} \in \mathcal{H}(b)\right) .
$$

It is now a well-known fact that the general theory of $\mathcal{H}(b)$-spaces splits into two cases, according to whether $b$ is an extreme point or a non-extreme point of the unit ball of $H^{\infty}$ (recall that, according to De Leeuw-Rudin's Theorem, $b$ is a non-extreme point of the closed unit ball of $H^{\infty}$ if and only if $\log (1-|b|) \in L^{1}(\mathbb{T})$, in particular every inner function $b=\Theta$ is an extreme point).

For example,

$$
\forall \lambda \in \mathbb{D}, k_{\lambda} \in \mathcal{H}(b) \Leftrightarrow b \text { is non - extreme, }
$$

(see [8, Theorem 23.23 and corollary 25.8]).

Furthermore from the above characterization of a non-extreme point it follows that, if $b$ is non-extreme, then there is an outer function $a$ such that $a(0)>0$ and $|a|^{2}+|b|^{2}=1$ a.e. on $\mathbb{T}$ [14]. The function $a$ is uniquely determined by $b$. We shall call $(a, b)$ an euclidian pair.The following result gives a useful characterization of $\mathcal{H}(b)$ in this case. 
Theorem 2.2 ([8], Theorem 23.8). Let $b$ be a non-extreme point of the closed unit ball of $H^{\infty}$, let $(a, b)$ be an euclidian pair and let $f \in H^{2}$. Then $f \in \mathcal{H}(b)$ if and only if $T_{\bar{b}} f \in T_{\bar{a}}\left(H^{2}\right)$. In this case, for $f_{1}, f_{2} \in \mathcal{H}(b)$ there exists a unique $f_{1}^{+}, f_{2}^{+} \in H^{2}$ such that $T_{\bar{b}} f_{i}=T_{\bar{a}} f_{i}^{+}$for $i=1,2$ and

$$
<f_{1}, f_{2}>_{b}=<f_{1}, f_{2}>_{2}+<f_{1}^{+}, f_{2}^{+}>_{2} \text {. }
$$

In particular, for each $f \in \mathcal{H}(b)$,

$$
\|f\|_{b}^{2}=\|f\|_{2}^{2}+\left\|f^{+}\right\|_{2}^{2}
$$

An important operator in the theory of model spaces is the compression of Toeplitz operators on $K_{\Theta}$ : for $\varphi \in L^{\infty}$ and $\Theta$ an inner function, one defines the truncated Toeplitz operator $A_{\varphi}^{\Theta}$ by

$$
\begin{array}{cccc}
A_{\varphi}^{\Theta}: & K_{\Theta} & \rightarrow & K_{\Theta} \\
f & \mapsto & A_{\varphi}^{\Theta} f=\mathbf{P}_{\Theta}\left(T_{\varphi} f\right),
\end{array}
$$

with $\mathbf{P}_{\Theta}$ the orthogonal projection of $H^{2}$ to $K_{\Theta}$. It turns out that when $\varphi$ is in $H^{\infty}$, then $K_{\Theta}$ is invariant for $T_{\bar{\varphi}}$ and the adjoint of the truncated Toeplitz operator with symbol $\varphi$ is $\left(A_{\varphi}^{\Theta}\right)^{\star}=T_{\bar{\varphi}, \Theta}[8$, Section 14.7]. More generally, for $\varphi \in H^{\infty}, \mathcal{H}(b)$ is invariant for $T_{\bar{\varphi}}$. We will denote by

$$
\begin{aligned}
& T_{\bar{\varphi}, b} \quad: \quad \mathcal{H}(b) \quad \rightarrow \quad \mathcal{H}(b) \\
& f \quad \mapsto \quad P_{+}(\bar{\varphi} f)
\end{aligned}
$$

and we have, $\left\|T_{\bar{\varphi}, b}\right\|_{L(\mathcal{H}(b))} \leq\|\varphi\|_{\infty}$ [8]. In particular, $\mathcal{H}(b)$ is invariant for the backward shift $S^{\star}=T_{\bar{z}}$. When $b$ is a non-extreme point of the closed unit ball of $H^{\infty}$, it follows from (1) and (2) that

$$
T_{\bar{\varphi}, b} k_{\lambda}=\overline{\varphi(\lambda)} k_{\lambda}, \lambda \in \mathbb{D} .
$$

\section{Compactness of $T_{\bar{\varphi}, b}$.}

Ahern and Clark [1] have given a necessary and sufficient condition for the truncated Toeplitz operator $A_{\varphi}^{\Theta}$ to be compact, when the symbol $\varphi$ is continuous on the boundary. See also an alternative proof by Garcia-RossWogen in [9]. The characterization of Ahern-Clark involves the notion of the spectrum of an inner function.

Recall that the spectrum of a function $b$ in the closed unit ball of $H^{\infty}$ [8, Section 5.2 and 22.6], denoted by $\sigma(b)$ is defined as follows

$$
\sigma(b)=\left\{\zeta \in \mathbb{T}: \lim _{\substack{z \rightarrow \zeta \\ z \in \mathbb{D}}} \inf |b(z)|<1\right\} \cup Z(b),
$$

where $Z(b)=\{\lambda \in \mathbb{D}: b(\lambda)=0\}$.

A generalization of Livsic-Moeller's result shows that $b$ and every element in $\mathcal{H}(b)$ can be analytically continued accross any $\operatorname{arc} I \subset \mathbb{T} \backslash \operatorname{clos}(\sigma(b))$, and $|b|=1$ on $I$ [8, Theorem 20.13].

In particular if $b=\Theta$ is a non constant inner function, and since $\Theta$ is unimodular a.e. on $\mathbb{t}$ then

$$
\sigma(\Theta)=\left\{\zeta \in \overline{\mathbb{D}}: \lim _{\substack{z \rightarrow \zeta \\ z \in \mathbb{D}}} \inf |\Theta(z)|=0\right\}=\operatorname{clos}(Z(\Theta)) \cup \operatorname{supp}(v),
$$

where $Z(\Theta)=\{\lambda \in \mathbb{D}: \Theta(\lambda)=0\}$ and $v$ is the measure representing the singular part of $\Theta$.

Now Ahern and Clark's result says:

Theorem 3.1 (Ahern-Clark, [1]). Let $\varphi \in C(\mathbb{T})$, then $A_{\varphi}^{\Theta}$ is compact if and only if $\varphi_{\mid \sigma(\Theta) \cap \mathbb{T}}=0$. 
The compactness property of the operators $T_{\bar{\varphi}, b}$ will depend on the boundary spectrum of $b$ and it is a consequence of the generalization of Ahern and Clark's result.

For this reason we begin by this generalization, and we study the compactness of the general operator $\mathbf{T}_{\bar{\varphi}, b}$ with $\varphi \in C(\mathbb{T})$, using the same technique used by Garcia, Ross and Wogen [9] to prove the Ahern-Clark result on compactness of $A_{\varphi}^{\Theta}$.

\subsection{Compactness of $\mathbf{T}_{\bar{\varphi}, b}$.}

Recall that the notation $\mathbf{T}_{\bar{\varphi}, b}$ represents the Toeplitz operator defined from $\mathcal{H}(b)$ into $H^{2}$.

Theorem 3.2. Let $b$ be a point of the closed unit ball of $H^{\infty}$ and let $\varphi \in C(\mathbb{T})$. Then the operator,

$$
\begin{array}{cccc}
\mathbf{T}_{\bar{\varphi}, b} \quad: \quad \mathcal{H}(b) & \rightarrow & H^{2} \\
f & \mapsto & P_{+}(\bar{\varphi} f)
\end{array},
$$

is compact if and only if $\varphi_{\mid \sigma(b) \cap \mathbb{T}}=0$.

Proof. $(\Leftarrow)$ Suppose that $\varphi_{\mid \sigma(b) \cap \mathbb{T}}=0$. Let $\varepsilon>0$ and pick $\psi \in C(\mathbb{T}) ; \psi=0$ on an open set containing $\operatorname{clos}(\sigma(b) \cap \mathbb{T})$ and $\|\psi-\varphi\|_{\infty}<\varepsilon$. Since $\left\|\mathbf{T}_{\bar{\varphi}, b}-\mathbf{T}_{\bar{\psi}, b}\right\|_{L\left(\mathcal{H}(b), H^{2}\right)} \leqslant\|\psi-\varphi\|_{\infty}<\varepsilon$, it suffices to show that $\mathbf{T}_{\bar{\psi}, b}$ is compact.

Let $K=\overline{\psi^{-1}(\mathbb{C} \backslash\{0\})}$ then $K \subset \mathbb{T} \backslash \operatorname{clos}(\sigma(b))$. And consider $\left(f_{n}\right)_{n}$ a sequence of $\mathcal{H}(b)$ such that $\left(f_{n}\right)_{n}$ weakly converges to zero.

We know that for each $\zeta \in K$, the function

$$
k_{\zeta}^{b}(z)=\frac{1-\overline{b(\zeta)} b(z)}{1-\bar{\zeta} z}
$$

belongs to $\mathcal{H}(b)$ and for every $f \in \mathcal{H}(b)$,

$$
f(\zeta)=<f, k_{\zeta}^{b}>_{b}
$$

and

$$
\left\|k_{\zeta}^{b}\right\|_{b}^{2}=\frac{1-|b(\zeta)|^{2}}{1-|\zeta|^{2}}=\left|b^{\prime}(\zeta)\right|
$$

(see [8, Theorem 21.1]).

In particular, since $\left(f_{n}\right)_{n}$ weakly converges to zero in $\mathcal{H}(b)$, we have $f_{n}(\zeta)=\left\langle f_{n}, k_{\zeta}^{b}>_{b} \rightarrow 0\right.$, as $n \rightarrow \infty$, and for every $n \in \mathbb{N},\left\|f_{n}\right\|_{b} \leq C$.

Therefore, since $b$ is analytic on a neighborhood of the compact set $K$ we obtain

$$
\forall \zeta \in K,\left|f_{n}(\zeta)\right|=\mid\left\langle f_{n}, k_{\zeta}^{b}>_{b}\left|\leq\left\|f_{n}\right\|_{b}\right|\right| k_{\zeta}^{b} \|_{b} \leq C \sup _{\zeta \in K} \sqrt{b^{\prime}(\zeta)}<\infty \quad .
$$

By the dominated convergence theorem, and using (4) it follows that

$$
\left\|\mathbf{T}_{\bar{\psi}, b} f_{n}\right\|_{2}^{2}=\left\|P_{+}\left(\bar{\psi} f_{n}\right)\right\|_{2}^{2} \leq\left\|\bar{\psi} f_{n}\right\|_{2}^{2}=\int_{\mathbb{T}}|\psi|^{2}\left|f_{n}\right|^{2} d \zeta=\int_{K}|\psi|^{2}\left|f_{n}\right|^{2} d \zeta \rightarrow 0 .
$$

whence $\mathbf{T}_{\bar{\psi}, b}$ is compact and therefore $\mathbf{T}_{\bar{\varphi}, b}$ is compact.

$(\Rightarrow)$ Suppose that $\varphi \in C(\mathbb{T}), \zeta \in \sigma(b) \cap \mathbb{T}$ and $\mathbf{T}_{\bar{\varphi}, b}$ is compact. Let

$$
F_{\lambda}(z)=\frac{1-|\lambda|^{2}}{1-|b(\lambda)|^{2}}\left|\frac{1-\overline{b(\lambda)} b(z)}{1-\bar{\lambda} z}\right|^{2},
$$

which is the square of the absolute value of the normalized reproducing kernel for $\mathcal{H}(b)$. Observe that 
$F_{\lambda}(z) \geq 0$.

Since $\zeta \in \sigma(b) \cap \mathbb{T}$ then there is a sequence $\lambda_{n}$ in $\mathbb{D}$ such that $\lambda_{n} \rightarrow \zeta$ and $\left|b\left(\lambda_{n}\right)\right| \rightarrow c$ with $c<1$ (by the definition of the spectrum of $b$ already mentioned). Suppose that $\zeta=e^{i \alpha}$ and note that if $|t-\alpha| \geq \delta$, then

$$
F_{\lambda_{n}}\left(e^{i t}\right) \leq C_{\delta} \frac{1-\left|\lambda_{n}\right|^{2}}{1-\left|b\left(\lambda_{n}\right)\right|^{2}}
$$

for some absolute constant $C_{\delta}>0$. Thus since $\left|b\left(\lambda_{n}\right)\right| \rightarrow c$ with $c<1$, we get that

$$
\sup _{|t-\alpha| \geq \delta} F_{\lambda_{n}}\left(e^{i t}\right) \rightarrow 0 \text { as } n \rightarrow \infty \text {. }
$$

Write,

$$
\begin{aligned}
& \varphi(\zeta) \frac{1}{2 \pi} \int_{-\pi}^{\pi} F_{\lambda_{n}}\left(e^{i t}\right) d t-\frac{1}{\left\|k_{\lambda_{n}}^{b}\right\|_{b}^{2}}<k_{\lambda_{n}}^{b}, \mathbf{T}_{\bar{\varphi}, b} k_{\lambda_{n}}^{b}>_{2} \\
& =\varphi(\zeta) \frac{1}{2 \pi} \int_{-\pi}^{\pi} F_{\lambda_{n}}\left(e^{i t}\right) d t-\frac{1}{\left\|k_{\lambda_{n}}^{b}\right\|_{b}^{2}}<k_{\lambda_{n}}^{b}, P_{+}\left(\bar{\varphi} k_{\lambda_{n}}^{b}\right)>_{2} \\
& =\varphi(\zeta) \frac{1}{2 \pi} \int_{-\pi}^{\pi} F_{\lambda_{n}}\left(e^{i t}\right) d t-\frac{1}{\left\|k_{\lambda_{n}}^{b}\right\|_{b}^{2}}<k_{\lambda_{n}}^{b}, \bar{\varphi} k_{\lambda_{n}}^{b}>_{2} \quad \text { (see [8, lemma 4.8]) } \\
& =\varphi(\zeta) \frac{1}{2 \pi} \int_{-\pi}^{\pi} F_{\lambda_{n}}\left(e^{i t}\right) d t-\frac{1}{\left\|k_{\lambda_{n}}^{b}\right\|_{b}^{2}} \frac{1}{2 \pi} \int_{-\pi}^{\pi} \varphi\left(e^{i t}\right)\left|k_{\lambda_{n}}^{b}\left(e^{i t}\right)\right|^{2} \\
& =\frac{1}{2 \pi} \int_{-\pi}^{\pi} \varphi(\zeta) F_{\lambda_{n}}\left(e^{i t}\right) d t-\frac{1}{2 \pi} \int_{-\pi}^{\pi} \varphi\left(e^{i t}\right) F_{\lambda_{n}}\left(e^{i t}\right) d t \\
& =\frac{1}{2 \pi} \int_{\pi}\left(\varphi(\zeta)-\varphi\left(e^{i t}\right)\right) F_{\lambda_{n}}\left(e^{i t}\right) d t \\
& =\frac{1}{2 \pi} \int_{|t-\alpha| \leq \delta}\left(\varphi(\zeta)-\varphi\left(e^{i t}\right)\right) F_{\lambda_{n}}\left(e^{i t}\right) d t+\frac{1}{2 \pi} \int_{|t-\alpha| \geq \delta}\left(\varphi(\zeta)-\varphi\left(e^{i t}\right)\right) F_{\lambda_{n}}\left(e^{i t}\right) d t .
\end{aligned}
$$

The first integral can be made small by the continuity of $\varphi$. Once $\delta>0$ is fixed the second term goes to zero since $\sup _{|t-\alpha| \geq \delta} F_{\lambda_{n}}\left(e^{i t}\right) \rightarrow 0$ as $n \rightarrow \infty$. In addition

$$
\begin{aligned}
\int_{-\pi}^{\pi} F_{\lambda_{n}}\left(e^{i t}\right) d t & =\frac{1-\left|\lambda_{n}\right|^{2}}{1-\left|b\left(\lambda_{n}\right)\right|^{2}} \int_{-\pi}^{\pi} \frac{\left|1-\overline{b\left(\lambda_{n}\right)} b\left(e^{i t}\right)\right|^{2}}{\left|1-\overline{\lambda_{n}} e^{i t}\right|^{2}} d t \\
& \geq \frac{1-\left|\lambda_{n}\right|^{2}}{1-\left|b\left(\lambda_{n}\right)\right|^{2}}\left(1-\left|b\left(\lambda_{n}\right)\right|\right)^{2} \int_{-\pi}^{\pi} \frac{1}{\left|1-\overline{\lambda_{n}} e^{i t}\right|^{2}} d t \\
& =\frac{\left(1-\left|\lambda_{n}\right|^{2}\right)\left(1-\left|b\left(\lambda_{n}\right)\right|\right)^{2}}{\left(1-\left|b\left(\lambda_{n}\right)\right|\right)\left(1+\left|b\left(\lambda_{n}\right)\right|\right)} \frac{1}{\left.|1-| \lambda_{n}\right|^{2}} \\
& =\frac{1-\left|b\left(\lambda_{n}\right)\right|}{1+\left|b\left(\lambda_{n}\right)\right|} \geq \frac{1-c}{2}>0 .
\end{aligned}
$$

Furthermore, on one hand

$$
\frac{\left\|k_{\lambda_{n}}^{b}\right\|_{2}}{\left\|k_{\lambda_{n}}^{b}\right\|_{b}} \leq 1
$$

And on the other hand the sequence $\left(\frac{k_{n}^{b}}{\left\|k_{\lambda_{n}}^{b}\right\|_{b}}\right)_{n}$ converges weakly to 0 , because $\left|\lambda_{n}\right| \rightarrow 1$ and $\left|b\left(\lambda_{n}\right)\right| \rightarrow c$ with $c<1$. Indeed, using that

$$
\left\|k_{\lambda_{n}}^{b}\right\|_{b}^{2}=\frac{1-\left|b\left(\lambda_{n}\right)\right|^{2}}{1-\left|\lambda_{n}\right|^{2}}
$$


We deduce that for $f \in H^{\infty} \cap \mathcal{H}(b)$,

$$
\left|<f, \frac{k_{\lambda_{n}}^{b}}{|| k_{\lambda_{n}}^{b} \|_{b}}>_{b}\right|=\frac{\left|f\left(\lambda_{n}\right)\right| \sqrt{1-\left|\lambda_{n}\right|^{2}}}{\sqrt{1-\left|b\left(\lambda_{n}\right)\right|^{2}}} \leq \frac{|| f \|_{\infty} \sqrt{1-\left|\lambda_{n}\right|^{2}}}{\sqrt{1-\left|b\left(\lambda_{n}\right)\right|^{2}}} \rightarrow 0 \quad \text { as } \quad n \rightarrow \infty .
$$

Furthermore, $H^{\infty} \cap \mathcal{H}(b)$ is dense in $\mathcal{H}(b)$, since for every $\lambda \in \mathbb{D}, k_{\lambda}^{b} \in H^{\infty} \cap \mathcal{H}(b)$. Thus the sequence $\frac{k_{\lambda_{n}}^{b}}{\left\|k_{\lambda_{n}}^{b}\right\|_{b}}$ converges weakly to 0 in $\mathcal{H}(b)$, with $\mathbf{T}_{\bar{\varphi}, b}$ considered compact. We deduce that

$$
\begin{aligned}
\frac{1}{\left\|k_{\lambda_{n}}^{b}\right\|_{b}^{2}}\left|<k_{\lambda_{n}}^{b}, \mathbf{T}_{\bar{\varphi}, b} k_{\lambda_{n}}^{b}>_{2}\right| & \leq \frac{\left\|k_{\lambda_{n}}^{b}\right\|_{2}}{\left\|k_{\lambda_{n}}^{b}\right\|_{b}} \frac{\left\|\mathbf{T}_{\bar{\varphi}, b} k_{\lambda_{n}}^{b}\right\|_{2}}{\left\|k_{\lambda_{n}}^{b}\right\|_{b}} \\
& \leq \frac{\left\|\mathbf{T}_{\bar{\varphi}, b} k_{\lambda_{n}}^{b}\right\|_{2}}{\left\|k_{\lambda_{n}}^{b}\right\|_{b}} \leq \frac{\left\|\mathbf{T}_{\bar{\varphi}, b} k_{\lambda_{n}}^{b}\right\|_{b}}{\left\|k_{\lambda_{n}}^{b}\right\|_{b}} \rightarrow 0 \text { as } n \rightarrow \infty .
\end{aligned}
$$

After all these computations, we see that

$$
\varphi(\zeta) \frac{1}{2 \pi} \int_{-\pi}^{\pi} F_{\lambda_{n}}\left(e^{i t}\right) d t-\frac{1}{\left\|k_{\lambda_{n}}^{b}\right\|_{b}^{2}}<k_{\lambda_{n}}^{b}, \mathbf{T}_{\bar{\varphi}, b} k_{\lambda_{n}}^{b}>_{2} \rightarrow 0 \text { as } n \rightarrow \infty .
$$

with

$$
\int_{-\pi}^{\pi} F_{\lambda_{n}}\left(e^{i t}\right) d t>0 \text { and } \frac{1}{\left\|k_{\lambda_{n}}^{b}\right\|_{b}^{2}}\left|<k_{\lambda_{n}}^{b}, \mathbf{T}_{\bar{\varphi}, b} k_{\lambda_{n}}^{b}>_{2}\right| \rightarrow 0 \text { as } n \rightarrow \infty .
$$

Finally, $\varphi(\zeta)=0$.

We now present consequences of this result.

\subsection{Compactness of $\boldsymbol{T}_{\bar{\varphi}, b}$}

Recall that the notation $T_{\bar{\varphi}, b}$ represents the Toeplitz operator defined from $\mathcal{H}(b)$ into itself.

Corollary 3.3. Let $b$ be a point of the closed unit ball of $H^{\infty}$ such that $m(\sigma(b) \cap \mathbb{T})>0$. Let $\varphi \in C(\mathbb{T}) \cap H^{\infty}$. Then the operator

$$
\begin{aligned}
& T_{\bar{\varphi}, b} \quad: \quad \mathcal{H}(b) \rightarrow \mathcal{H}(b) \\
& f \quad \mapsto P_{+}(\bar{\varphi} f),
\end{aligned}
$$

is compact if and only if $\varphi=0$.

Proof. Assume that $T_{\bar{\varphi}, b}$ is compact. Then $\mathbf{T}_{\bar{\varphi}, b}$ is also compact and by Theorem 3.2,

$$
\varphi(\sigma(b) \cap \mathbb{T})=0 .
$$

However, since $\varphi \in H^{\infty}$ with $m(\sigma(b) \cap \mathbb{T})>0$ then $\varphi=0$.

Corollary 3.4. Let $b$ be a point of the closed unit ball of $H^{\infty}$. The following assertions are equivalent:

1. $\exists \varphi \in H^{\infty} \cap C(\mathbb{T}), \varphi \neq 0$ such that $T_{\bar{\varphi}, b}$ is compact.

2. $m(\sigma(b) \cap \pi)=0$. 
Proof. (2) $\Rightarrow(1)$ First note that (2) implies that $b$ is an inner function. Indeed, assume on the contrary that $b$ is not inner. Then, the set

$$
E=\{\zeta \in \mathbb{T}:|b(\zeta)| \neq 1\}
$$

has a positive Lebesgue mesure. Moreover, it turns out that $E \subset \operatorname{clos}(\sigma(b)) \cap \mathbb{T}$. Indeed, if $\zeta \in \mathbb{T} \backslash \operatorname{clos}(\sigma(b))$ then $b$ admits an analytic continuation across a neighborhood $D(\zeta, r)=\{w:|w-\zeta|<r\}$ of $\zeta$ with $|b| \equiv 1$ on the $\operatorname{arc} D(\zeta, r) \cap \mathbb{\pi}$.

In particular, $|b(\zeta)|=1$ and $\zeta \in \mathbb{T} \backslash E$. We deduce that

$$
0<m(E) \leq m(\operatorname{clos}(\sigma(b)) \cap \mathbb{T})) .
$$

Now according to Rudin's theorem, we can find a function $\varphi \in \mathcal{A}(\mathbb{D})=\operatorname{Hol}(\mathbb{D}) \cap C(\mathbb{D}), \varphi \neq 0$ such that $\varphi(\sigma(b) \cap \mathbb{T})=0$. Then we apply Ahern-Clark's result (see Theorem 3.1) to get that $T_{\bar{\varphi}, b}=\left(A_{\varphi}^{b}\right)^{\star}$ is compact. (1) $\Rightarrow$ (2) Follows from Corollary 3.3.

In the case where $b$ is a non-extreme point of the closed unit ball of $H^{\infty}$, we can get a more general result without the hypothesis that the symbol $\varphi$ is continuous.

Theorem 3.5. Let $b$ be a non-extreme point of the closed unit ball of $H^{\infty}$ and let $\varphi \in H^{\infty}$. Then the operator

$$
\begin{aligned}
& T_{\bar{\varphi}, b}: \mathcal{H}(b) \rightarrow \mathcal{H}(b) \\
& f \quad \mapsto \quad P_{+}(\bar{\varphi} f) .
\end{aligned}
$$

is compact if and only if $\varphi=0$.

Proof. Let $a$ be the unique outer function such that $(a, b)$ is an euclidian pair. Note that since $b$ is nonextreme then $k_{z} \in \mathcal{H}(b)$, for all $z \in \mathbb{D}$ (see (2)).

Suppose that $T_{\bar{\varphi}, b}$ is compact. Notice that for every $\left(\lambda_{n}\right)_{n} \subset \mathbb{D}$ such that $\left|\lambda_{n}\right| \rightarrow 1$, the sequence $\left(\frac{k_{\lambda_{n}}}{\left\|k_{\lambda_{n}}\right\|_{b}}\right)_{n}$ converges weakly to 0 in $\mathcal{H}(b)$.

Indeed, let $f \in \mathcal{H}(b)$ such that $f$ and $f^{+} \in H^{\infty}$. Recall that $f^{+}$is defined in Theorem 2.2. Then, using that

$$
T_{\bar{b}} k_{\lambda_{n}}=\overline{b\left(\lambda_{n}\right)} k_{\lambda_{n}}=T_{\bar{a}}\left(\frac{\overline{b\left(\lambda_{n}\right)}}{\overline{a\left(\lambda_{n}\right)}} k_{\lambda_{n}}\right) \text { (see (3)), }
$$

we see that

$$
k_{\lambda_{n}}^{+}=\frac{\overline{b\left(\lambda_{n}\right)}}{\overline{a\left(\lambda_{n}\right)}} k_{\lambda_{n}} .
$$

Whence by Theorem 2.2, we have

$$
\begin{aligned}
\left\langle f, \frac{k_{\lambda_{n}}}{\left\|k_{\lambda_{n}}\right\|_{b}}>_{b}\right. & =\left\langle f, \frac{k_{\lambda_{n}}}{\left\|k_{\lambda_{n}}\right\|_{b}}>_{2}+\left\langle f^{+}, \frac{k_{\lambda_{n}}^{+}}{\left\|k_{\lambda_{n}}\right\|_{b}}\right\rangle_{2}\right. \\
& =\left(f\left(\lambda_{n}\right)+\frac{b\left(\lambda_{n}\right)}{a\left(\lambda_{n}\right)} f^{+}\left(\lambda_{n}\right)\right) \frac{1}{\left\|k_{\lambda_{n}}\right\|_{b}} .
\end{aligned}
$$

On the other hand, it is known that in the non-extreme case:

$$
\left\|k_{\lambda_{n}}\right\|_{b}^{2}=\frac{1}{1-\left|\lambda_{n}\right|^{2}}\left(1+\frac{\left|b\left(\lambda_{n}\right)\right|^{2}}{\left|a\left(\lambda_{n}\right)\right|^{2}}\right)
$$

(see [8, Corollary 23.25]). Hence

$$
\frac{\left|b\left(\lambda_{n}\right)\right|^{2}}{\left.\left|a\left(\lambda_{n}\right)\right|^{2}|| k_{\lambda_{n}}\right|_{b} ^{2}}=\frac{\left(1-\left|\lambda_{n}\right|^{2}\right)\left|b\left(\lambda_{n}\right)\right|^{2}}{\left|a\left(\lambda_{n}\right)\right|^{2}+\left|b\left(\lambda_{n}\right)\right|^{2}} \leq 1-\left|\lambda_{n}\right|^{2} .
$$


Using this inequality and the inequality

$$
\left\|k_{\lambda_{n}}\right\|_{b}^{2} \geq \frac{1}{1-\left|\lambda_{n}\right|^{2}}
$$

we deduce that

$$
\begin{aligned}
\left|<f, \frac{k_{\lambda_{n}}}{\left\|k_{\lambda_{n}}\right\|_{b}}>_{b}\right| & \leq\left(\left|f\left(\lambda_{n}\right)\right|+\left|f^{+}\left(\lambda_{n}\right)\right|\right) \sqrt{1-\left|\lambda_{n}\right|^{2}} \\
& \leq\left(|| f\left\|_{\infty}+|| f^{+}\right\|_{\infty}\right) \sqrt{1-\left|\lambda_{n}\right|^{2}} \rightarrow 0 \text { as } n \rightarrow \infty .
\end{aligned}
$$

Furthermore, the set $\left\{f \in \mathcal{H}(b) ; f \& f^{+} \in H^{\infty}\right\}$ is dense in $\mathcal{H}(b)$, since $\left\{k_{\lambda}^{b} ; \lambda \in \mathbb{D}\right\} \subset\left\{f \in \mathcal{H}(b) ; f \& f^{+} \in\right.$ $\left.H^{\infty}\right\}$. Indeed, for every $\lambda \in \mathbb{D}$,

$$
\left(k_{\lambda}^{b}\right)^{+}=\left(k_{\lambda}-\overline{b(\lambda)} b k_{\lambda}\right)^{+}=k_{\lambda}^{+}-\overline{b(\lambda)}\left(b k_{\lambda}\right)^{+}=\overline{b(\lambda)} a k_{\lambda} \in H^{\infty},
$$

with

$$
\left(b k_{\lambda}\right)^{+}=\frac{k_{\lambda}}{\overline{a(\lambda)}}-a k_{\lambda}
$$

(see [8, Theorem 23.23]).

Hence, the sequence $\left(\frac{k_{\lambda_{n}}}{\left\|k_{\lambda_{n}}\right\|_{b}}\right)_{n}$ converges weakly to 0 in $\mathcal{H}(b)$.

Now by compactness of $T_{\bar{\varphi}, b}$ it follows that for every $\left(\lambda_{n}\right)_{n} \subset \mathbb{D}$ such that $\left|\lambda_{n}\right| \rightarrow 1$,

$$
\left\|T_{\bar{\varphi}, b} \frac{k_{\lambda_{n}}}{\left\|k_{\lambda_{n}}\right\|_{b}}\right\|_{b} \rightarrow 0 \quad \text { as } \quad n \rightarrow \infty
$$

But $T_{\bar{\varphi}, b} k_{\lambda_{n}}=\overline{\varphi\left(\lambda_{n}\right)} k_{\lambda_{n}}$ (see (3)). Thus (5) is equivalent to

$$
\forall\left(\lambda_{n}\right)_{n} \subset \mathbb{D} ; \lim _{n \rightarrow \infty}\left|\lambda_{n}\right|=1,\left|\varphi\left(\lambda_{n}\right)\right| \rightarrow 0 .
$$

Which implies that $\varphi=0$.

The proof of Theorem 3.5 obviously doesn't work in the case when $b$ is an extreme point of the closed unit ball of $H^{\infty}$, since in that case, the Cauchy kernels $k_{\lambda}$ do not belong to $\mathcal{H}(b)$ when $b(\lambda) \neq 0, \lambda \in \mathbb{D}$.

\section{Hypercyclicity of $\boldsymbol{T}_{\bar{\varphi}, b}$}

\subsection{Hypercyclic and frequently hypercyclic operators}

Let $X$ be a complex infinite-dimensional separable Banach space. An operator $T \in L(X)$ is said to be hypercyclic if there is some vector $x \in X$ such that the orbit

$$
O(x, T):=\left\{T^{n}(x) ; n \in \mathbb{N}\right\}
$$

is dense in $X$. Such a vector $x$ is said to be hypecyclic for $T$, and the set of all hypercyclic vectors for $T$ is denoted by $H C(T)$.

Moreover we say that $T$ is frequently hypercyclic, if there exists a vector $x \in X$ such that for every nonempty open subset $U$ of $X$, the set $N(x, U)=\left\{n \geq 0 ; T^{n}(x) \in U\right\}$ of instants when the iterates of $x$ under $T$ visit $U$ has positive lower density, i.e.

$$
\underline{\operatorname{dens}}(N(x, U))=\lim _{N \rightarrow \infty} \inf \frac{\operatorname{card}(N(x, U) \cap[1, N])}{N}>0 .
$$


We refer the reader to the recent book [4] for more information on these topics.

Frequent hypercyclicity is a much stronger notion than hypercyclicity, and some operators are hypercyclic without being frequently hypercyclic: an example is the Bergman backward shift [3].

Let us complete this section by recalling two criterions for hypercyclicity and frequent hypercyclicity that we will use to study the hypercyclicity properties of the Toeplitz operator $T_{\bar{\varphi}, b}$.

We start with the Godefroy-Shapiro Criterion [11], according to which a bounded operator having a large supply of eigenvectors associated to eigenvalues of modulus strictly larger than 1 and strictly smaller than 1 is hypercyclic.

Theorem 4.1 (Godefroy-Shapiro Criterion, [11]). Let $T \in L(X)$. Suppose that $\bigcup_{|\lambda|<1} \operatorname{Ker}(T-\lambda)$ and $\bigcup_{|\lambda|>1} \operatorname{Ker}(T-\lambda)$ both span a dense subspace of $X$. Then $T$ is hypercyclic.

Then it was shown by S. Grivaux that an operator $T$ which has "sufficiently many" eigenvectors associated to eigenvalues of modulus 1 is automatically frequently hypercyclic.

Theorem 4.2 (S. Grivaux, [12]). Let $T$ be a bounded operator on X. Suppose that there exists a sequence $\left(u_{i}\right)_{i \geq 1}$ of vectors of $X$ having the following properties:

1. For each $i \geq 1, u_{i}$ is an eigenvector of $T$ associated to an eigenvalue $\lambda_{i}$ of $T$ with $\left|\lambda_{i}\right|=1$ and the $\lambda_{i}$ 's all distinct;

2. $\operatorname{span}\left[u_{i} ; i \geq 1\right]$ is dense in $X$;

3. For any $i \geq 1$ and any $\varepsilon>0$, there exists an $n \neq i$ such that $\left\|u_{n}-u_{i}\right\|<\varepsilon$.

Then $T$ is frequently hypercyclic (in particular hypercyclic).

It is also a natural question, given a family of hypercyclic operators to ask if they have a common hypercyclic vector. The following result gives a sufficient condition for a family of multiples of an operator to have a dense $G_{\delta}$-set of common hypercyclic vectors.

Theorem 4.3 (Shkarin, [17]). Let $X$ be a separable Fréchet space, $T \in L(X), 0 \leq a<c \leq \infty$. Assume also that for all $\alpha, \beta \in \mathbb{R}$ such that $a<\alpha<\beta<c$ there exists a dense subset $E$ of $X$ and a map $S: E \longrightarrow E$ such that $T S x=x, \alpha^{-n} T^{n} x \rightarrow 0$ and $\beta^{n} S^{n} x \rightarrow 0$ for each $x \in E$. Then

$$
\cap H C\left(\lambda T: c^{-1}<|\lambda|<a^{-1}\right),
$$

is a dense $G_{\delta}$-set in $X$.

We finish by giving an example of a hypercyclic Toeplitz operator.

Rolewicz's result [4] in 1960, says that the operator $\lambda S^{\star}=T_{\lambda \bar{z}}: H^{2} \rightarrow H^{2}$ for every $\lambda \in \mathbb{C},|\lambda|>1$, is hypercyclic, this was shown using Kitai's Criterion (a particular case of the Hypercyclity Criterion) [10]. This result of Rolewicz was generalized by Godefroy-Shapiro [11] in 1991.

Theorem 4.4 (Godefroy-Shapiro). Let $\varphi \in H^{\infty}$. The operator $T_{\bar{\varphi}}: H^{2} \rightarrow H^{2}$ is hypercyclic if and only if $\varphi$ is non-constant and $\varphi(\mathbb{D}) \cap \mathbb{\mathbb { T }} \neq \emptyset$. 


\subsection{Hypercyclity of $\boldsymbol{T}_{\bar{\varphi}, b}$.}

Following the approach of Godefroy-Shapiro, we generalize Theorem 4.4 to operators $T_{\bar{\varphi}, b}$ when $b$ is a nonextreme point of the closed unit ball of $H^{\infty}$ and $\varphi \in H^{\infty}$, even we get a better result. And according to this generalization we noticed that in the non-extreme case, for every $|\lambda|>1$ the operator $\lambda X_{b}=\lambda T_{\bar{z}, b}$ is frequently hypercyclic (in particular hypercyclic). On the contrary when $b$ is extreme, $\lambda X_{b}$ is never hypercyclic.

Theorem 4.5. Let $b$ be a non-extreme point of the closed unit ball of $H^{\infty}$ and let $\varphi \in H^{\infty}$. Then the operator

$$
\begin{array}{rllc}
T_{\bar{\varphi}, b}: \quad \mathcal{H}(b) & \rightarrow & \mathcal{H}(b) \\
& f & \mapsto & T_{\bar{\varphi}, b} f=P_{+}(\bar{\varphi} f) .
\end{array}
$$

is frequently hypercyclic if and only if $\varphi$ is non-constant and $\varphi(\mathbb{D}) \cap \mathbb{T} \neq \emptyset$.

Proof. Let $\varphi$ be a non constant analytic function on $\mathbb{D}$ and assume that $\varphi(\mathbb{D}) \cap \mathbb{T} \neq \emptyset$. Let $\zeta_{0} \in \varphi(\mathbb{D}) \cap \mathbb{T}$. Since $\varphi(\mathbb{D})$ is an open set of $\mathbb{C}$ (open mapping theorem), there exists $r>0$ such that $D\left(\zeta_{0}, r\right) \subset \varphi(\mathbb{D})$ and let $\Gamma$ be a closed arc contained in $D\left(\zeta_{0}, r\right) \cap \mathbb{\mathbb { C }} \subset \varphi(\mathbb{D})$. Consider an exhaustive sequence of compacts $\left(K_{n}\right)_{n}$ associated to $\mathbb{D}$. Then

$$
\varphi^{-1}(\Gamma)=\bigcup_{n \geq 1} \varphi^{-1}(\Gamma) \cap K_{n},
$$

since $\varphi^{-1}(\Gamma)$ is uncountable, there is, indeed, $n_{0} \geq 1$ such that $\operatorname{Card}\left(\varphi^{-1}(\Gamma) \cap K_{n_{0}}\right)=+\infty$. We can therefore construct a sequence $\left(\lambda_{n}\right)_{n}$ in $\varphi^{-1}(\Gamma) \cap K_{n_{0}}$ with $\lambda_{n} \neq \lambda_{\ell}, n \neq \ell$. Now $\lambda_{n} \in K_{n_{0}}$-compact, so there is a subsequence $\left(\lambda_{n_{\ell}}\right)_{\ell}$ such that $\lambda_{n_{\ell}} \rightarrow \lambda \in K_{n_{0}} \subset \mathbb{D}, \ell \rightarrow+\infty$. Since $b$ is non-extreme, reproducing kernels of $H^{2}, k_{\lambda}$ for all $\lambda \in \mathbb{D}$, are elements of $\mathcal{H}(b)$ and they are eigenvectors of $T_{\bar{\varphi}, b}$, of eigenvalues $\overline{\varphi(\lambda)}$ (see (3)).Thus the sequence of reproducing kernels of $H^{2}$ associated to the subsequence $\left(\lambda_{n_{\ell}}\right)_{\ell}$ will be dense in $\mathcal{H}(b)$ (since $\lambda$ is the unique accumulation point of this subsequence):

$$
\operatorname{Span}\left(k_{\lambda_{n_{\ell}}}: n_{\ell}\right)=\mathcal{H}(b)
$$

and $\left|\varphi\left(\lambda_{n_{\ell}}\right)\right|=1$. Moreover this sequence of eigenvectors satisfies the property of "continuity" which is the third condition of Grivaux's criterion (see Theorem 4.2) because the application $\mu \rightarrow k_{\mu}$ is continuous and the subsequence is convergent. Hence the Toeplitz operator $T_{\bar{\varphi}, b}$ is frequently hypercyclic.

Conversely, assume that $T_{\bar{\varphi}, b}$ is frequently hypercyclic then it is in particular hypercyclic ( so that $\varphi$ is certainly non constant). And assume that $\varphi(\mathbb{D}) \cap \mathbb{T}=\emptyset$. Since $\mathbb{D}$ is connected with $\varphi$ continuous on $\mathbb{D}, \varphi(\mathbb{D})$ is connected, hence $\varphi(\mathbb{D}) \subset \mathbb{D}$ or $\varphi(\mathbb{D}) \subset \mathbb{C} \backslash \overline{\mathbb{D}}$. If $\varphi(\mathbb{D}) \subset \mathbb{D}$ then $\forall z \in \mathbb{D},|\varphi(z)|<1$, it implies that $\|\varphi\|_{\infty} \leq 1$ and $\left\|T_{\bar{\varphi}, b}\right\| \leq\|\varphi\|_{\infty} \leq 1$, whence $T_{\bar{\varphi}, b}$ is non-hypercyclic (absurd). If $\varphi(\mathbb{D}) \subset \mathbb{C} \backslash \mathbb{D}$ then $\forall z \in \mathbb{D},|\varphi(z)|>1$. In this case, $\frac{1}{\varphi} \in H^{\infty}$ and $T_{\frac{1}{\bar{\varphi}}, b}$ is non-hypercyclic since $\left\|T_{\frac{1}{\bar{\varphi}}, b}\right\| \leq\left\|\frac{1}{\bar{\varphi}}\right\|_{\infty} \leq 1$. Seeing that, $T_{\bar{\varphi}, b} T_{\frac{1}{\bar{\phi}}, b}=T_{\frac{1}{\bar{\varphi}}, b} T_{\bar{\varphi}, b}=I$, then $T_{\frac{1}{\bar{\phi}}, b}=\left(T_{\bar{\varphi}, b}\right)^{-1}$, consequently $T_{\bar{\varphi}, b}$ is non-hypercyclic( indeed an invertible operator is hypercyclic if and only if its inverse is hypercyclic [4, page 3] ). We get also a contradiction.

Remark 4.6. Note that when $b=0$, we recover Theorem 4.4 of Godefroy and Shapiro.

In the particular case when $\varphi(z)=z$, corresponding to operator $X_{b}: \mathcal{H}(b) \rightarrow \mathcal{H}(b), X_{b}(f)=S^{\star} f$, we get the following result:

Corollary 4.7. Let $b$ be a non-extreme point of the closed unit ball of $H^{\infty}$. Let the operator

$$
\begin{aligned}
X_{b}: \quad: \mathcal{H}(b) & \rightarrow \mathcal{H}(b) \\
f & \mapsto S^{\star} f
\end{aligned}
$$

For all $|\lambda|>1, \lambda X_{b}$ is frequently hypercyclic (in particular hypercyclic). 
As we saw in the previous Corollary, for all $|\lambda|>1, \lambda X_{b}$ is hypercyclic, so this naturally raises the question of finding a common hypercyclic vector for $\left(\lambda X_{b}\right)_{|\lambda|>1}$. We will apply Shkarin's Theorem 4.3 but we need to introduce another operator on $\mathcal{H}(b)$.

It is well known that $\mathcal{H}(b)$ is invariant under the unilateral forward shift operator $S$ if and only if $b$ is non-extreme [8, Corollary 20.20]. In that case, the mapping

$$
\begin{aligned}
S_{b}: \quad \mathcal{H}(b) & \rightarrow \mathcal{H}(b) \\
f & \mapsto S f=z f
\end{aligned} .
$$

gives a well-defined operator. Moreover $S_{b}$ is bounded on $\mathcal{H}(b)$ with $\left\|S_{b}\right\|=\sqrt{1+|a(0)|^{-2}}\left\|S^{\star} b\right\|_{b}^{2}$ (see [8, Section 24.1]) In particular, we see that except in the case when $b=0$ (corresponding to $\mathcal{H}(b)=H^{2}$ ), the operator $S_{b}$ has a norm strictly greater than 1 .

Theorem 4.8. Let $b$ be a non-extreme point of the closed unit ball of $H^{\infty}$, and let

$$
\begin{aligned}
X_{b} \quad: \quad \mathcal{H}(b) & \rightarrow \mathcal{H}(b) \\
f & \mapsto S^{\star} f
\end{aligned} .
$$

Then,

$$
\mathcal{G}=\bigcap H C\left(\lambda X_{b} ;|| S_{b} \|<|\lambda|<\infty\right)
$$

is a dense $G_{\delta}$-set of $\mathcal{H}(b)$.

Proof. We would like to apply Shkarin's Theorem 4.3 with $a=0, c=\left\|S_{b}\right\|^{-1}$ and $E=\mathcal{P}$, with $\mathcal{P}$ the set of analytic polynomials, dense in $\mathcal{H}(b)$ [8, Theorem 23.13]. Let

$$
S_{b}: \mathcal{P} \longrightarrow \mathcal{P} ; S_{b} p=z p .
$$

It is clear that $X_{b} S_{b}=I$. For all $0<\alpha<\beta<\left\|S_{b}\right\|^{-1}$, and for all $p \in \mathcal{P}$, we have on one hand $\alpha^{-n} X_{b}^{n} p \rightarrow 0$ as $n \rightarrow$ $\infty$, since from a certain rank $n_{0}=\operatorname{deg}(p)+1, X_{b}^{n_{0}} p=0$, and on the other hand, $\left\|\beta^{n} S_{b}^{n} p\right\|_{b} \leq\left(\beta\left\|S_{b}\right\|\right)^{n}\|p\|_{b} \rightarrow 0$ as $n \rightarrow \infty$. Hence, using Theorem 4.3, we conclude that $\mathcal{G}$ is a dense $G_{\delta}$-set of $\mathcal{H}(b)$.

Remark 4.9. It remains the question of whether we can replace in the previous Theorem the lower bound

$$
\left\|S_{b}\right\|<|\lambda| \text { by } 1<|\lambda| \text {. }
$$

In other word, is the set

$$
\bigcap H C\left(\lambda X_{b} ; 1<|\lambda|<\infty\right)
$$

a dense $G_{\delta}$-set of $\mathcal{H}(b)$ ?

In the case where $b$ is extreme, the operator $X_{b}$ is no longer hypercyclic, which shows a significant difference in the $\mathcal{H}(b)$ space theory following that $\log (1-|b|)$ is integrable or not on $\mathbb{t}$. The proof of this result requires basic facts on the spectrum of hypercyclic operators, which we now briefly recall.

Let $X$ be a complex Banach space, and let $T \in \mathcal{L}(X)$ be hypercyclic. Then $\sigma_{p}\left(T^{\star}\right)=\emptyset$ and every connected component of the spectrum of $T$ intersects the unit circle (see [4, Page 11]).

Theorem 4.10. Let $b$ be an extreme point of the closed unit ball of $H^{\infty}$ and let

$$
\begin{aligned}
X_{b}: \mathcal{H}(b) & \rightarrow \mathcal{H}(b) \\
f & \mapsto S^{\star} f
\end{aligned}
$$

Then for every complex number $\lambda, \lambda X_{b}$ is not hypercyclic.

Proof. For all $|\lambda| \leq 1,|| \lambda X_{b} \| \leq 1$ hence $\lambda X_{b}$ is not hypercyclic. Now take $\lambda \in \mathbb{C},|\lambda|>1$. By [8, Corollary 26.3], we have

$$
\sigma_{p}\left(\bar{\lambda} X_{b}^{\star}\right)=\bar{\lambda} \sigma_{p}\left(X_{b}^{*}\right)=\{\bar{\lambda} \beta: \beta \in \mathbb{D} \text { and } b(\beta)=0\} \text {. }
$$


By the preceding equality, we notice that, if $b$ has a Blaschke factor then $\sigma_{p}\left(\bar{\lambda} X_{b}^{\star}\right) \neq \emptyset$, and thus $\lambda X_{b}$ is not hypercyclic. Now if $b$ does not admit a Blaschke factor we get from [8, Corollary 26.4] that $\sigma\left(X_{b}\right) \subset \mathbb{T}$. That implies, since $|\lambda|>1, \sigma\left(\lambda X_{b}\right) \cap \mathbb{T}=\left(\lambda \sigma\left(X_{b}\right)\right) \cap \mathbb{T}=\emptyset$. Therefore one of the connected component of $\sigma\left(\lambda X_{b}\right)$ do not intersect the unit circle, hence $\lambda X_{b}$ is not hypercyclic. We conclude that for every complex number $\lambda, \lambda X_{b}$ is not hypercyclic.

In the case when $b$ is extreme, it has not been possible to reach the non-hypercyclicity of the Toeplitz operator $T_{\bar{\varphi}, b}$. However we give a necessary (but not sufficient) condition for such an operator to be non-hypercyclic.

Proposition 4.11. Let $b$ be an extreme point of the closed unit ball of $H^{\infty}$, and let $\varphi \in H^{\infty}$. If the Toeplitz operator $T_{\bar{\varphi}, b}$ is hypercyclic then $\sigma_{p}\left(T_{\bar{\varphi}, b}\right)=\emptyset$.

Proof. From [18] it follows that $T_{\bar{\varphi}, b}$ is complex symmetric, and therefore, if it has an eigenvalue, the adjoint also has an eigenvalue and in this case $T_{\bar{\varphi}, b}$ is not hypercyclic.

In particular, if $b$ has a blaschke factor, then $T_{\bar{\varphi}, b}$ is not hypercyclic, as shown in the following result.

Corollary 4.12. If $\lambda \in \mathbb{D}$ such that $b(\lambda)=0$ then $T_{\bar{\varphi}, b}$ is not hypercyclic

Proof. Suppose that $\lambda \in \mathbb{D}$ such that $b(\lambda)=0$, then $k_{\lambda}=k_{\lambda}^{b} \in \mathcal{H}(b)$. Moreover $T_{\bar{\varphi}, b} k_{\lambda}=\overline{\varphi(\lambda)} k_{\lambda}$ (see (3)). Hence $\overline{\varphi(\lambda)} \in \sigma_{p}\left(T_{\bar{\varphi}, b}\right)$. Thus by Proposition 4.11, $T_{\bar{\varphi}, b}$ is not hypercyclic.

Remark 4.13. It turns out that the necessary condition in proposition 4.11 is not sufficient. Indeed, if $b$ has no Blaschke factor, then $\sigma_{p}\left(X_{b}\right)$ is empty, though by Theorem 4.10, we know that $X_{b}$ is not hypercyclic.

Remark 4.14. Notice that

$$
\sigma_{p}\left(T_{\bar{\varphi}, b}\right)=\emptyset \Leftrightarrow \forall \lambda \in \mathbb{C}, K_{(\varphi-\lambda)_{i}} \cap \mathcal{H}(b)=\{0\},
$$

with $\varphi-\lambda=(\varphi-\lambda)_{i}(\varphi-\lambda)_{e}$, and $(\varphi-\lambda)_{i}$ and $(\varphi-\lambda)_{e}$ are respectively the inner and outer part of $\varphi-\lambda$.

Proof. Let $\lambda \in \mathbb{C}$. Then $\bar{\lambda} \in \sigma_{p}\left(T_{\bar{\varphi}, b}\right)$ if and only if there exists $f \in \mathcal{H}(b), f \neq 0$ such that

$$
T_{\bar{\varphi}, b} f=T_{\bar{\varphi}} f=\bar{\lambda} f
$$

This equation is equivalent to

$$
T_{\overline{\varphi-\lambda}} f=0 \Leftrightarrow T_{\overline{(\varphi-\lambda)})_{e}(\overline{\varphi-\lambda})_{i}} f=0 \Leftrightarrow T_{(\overline{\varphi-\lambda})_{i}} f=0,
$$

because when $a$ is outer $T_{\bar{a}}$ is one-to-one. The last equation is equivalent to

$$
f \in K_{(\varphi-\lambda)_{i}} \cap \mathcal{H}(b),
$$

where $K_{(\varphi-\lambda)_{i}}$ denotes the model space associated to $(\varphi-\lambda)_{i}$. Thus $\bar{\lambda} \in \sigma_{p}\left(T_{\bar{\varphi}, b}\right)$ if and only if $K_{(\varphi-\lambda)_{i}} \cap \mathcal{H}(b) \neq$ $\{0\}$.

Remark 4.15. If $b$ is extreme and outer, then $\sigma_{p}\left(T_{\bar{\varphi}, b}\right)=\emptyset$.

Proof. Let $\lambda \in \mathbb{C}$.

If $(\varphi-\lambda)_{i} \equiv$ cte then $K_{(\varphi-\lambda)_{i}}=\{0\}$ (see [8, Theorem 18.2]), which implies that $K_{(\varphi-\lambda)_{i}} \cap \mathcal{H}(b)=\{0\}$. On the other hand if $(\varphi-\lambda)_{i} \neq c t e$, and if $f \in K_{(\varphi-\lambda)_{i}}$ then $f$ is not a cyclic vector for $S^{\star}$ (since span $\left\{S^{\star} n f\right.$ : $n \geq 0\} \subset K_{(\varphi-\lambda)_{i}} \neq H^{2}$ because $S^{\star} K_{(\varphi-\lambda)_{i}} \subset K_{(\varphi-\lambda)_{i}}$, see section 2.1 and [8, Section 1.10]). Using [8, Theorem 25.17], it implies $f \in K_{\Theta}$ where $\Theta=b_{i}$ is the inner part of $b$. But since $b$ is considered outer, then $b_{i} \equiv c t e$, 
thus $K_{\Theta}=\{0\}$. Hence $K_{(\varphi-\lambda)_{i}} \cap \mathcal{H}(b)=\{0\}$.

Which gives by Remark 4.14 that $\sigma_{p}\left(T_{\bar{\varphi}, b}\right)=\emptyset$.

Remark 4.16. In the case where $b$ is extreme and outer, it would be interesting to know if $T_{\bar{\varphi}, b}$ is hypercyclic or not.

Acknowledgements: The author deeply thank the anonymous referee for his/her valuable remarks and suggestions which improved the quality of this paper. The author would like also to warmly thank Emmanuel Fricain for his support and for several valuable discussions.

The author was supported by the Laboratory CEMPI (Centre Européen pour les Mathématiques, la Physique et leurs interactions).

\section{References}

[1] P. R. Ahern and D. N. Clark. On functions orthogonal to invariant subspaces. Acta Math., 124:191-204, 1970.

[2] Anton Baranov and Andrei Lishanskii. Hypercyclic Toeplitz operators. Results Math., 70(3-4):337-347, 2016.

[3] Frédéric Bayart and Sophie Grivaux. Hypercyclicity and unimodular point spectrum. J. Funct. Anal., 226(2):281-300, 2005.

[4] Frédéric Bayart and Étienne Matheron. Dynamics of linear operators, volume 179 of Cambridge Tracts in Mathematics. Cambridge University Press, Cambridge, 2009.

[5] Arlen Brown and P. R. Halmos. Algebraic properties of Toeplitz operators. J. Reine Angew. Math., 213:89-102, $1963 / 1964$.

[6] Louis de Branges and James Rovnyak. Canonical models in quantum scattering theory. In Perturbation Theory and its Applications in Quantum Mechanics (Proc. Adv. Sem. Math. Res. Center, U.S. Army, Theoret. Chem. Inst., Univ. of Wisconsin, Madison, Wis., 1965), pages 295-392. Wiley, New York, 1966.

[7] Louis de Branges and James Rovnyak. Square summable power series. Holt, Rinehart and Winston, New York-Toronto, Ont.-London, 1966.

[8] Emmanuel Fricain and Javad Mashreghi. The theory of $\mathcal{H}(b)$ spaces. Vol. 2, volume 21 of New Mathematical Monographs. Cambridge University Press, Cambridge, 2016.

[9] Stephan Ramon Garcia, William T. Ross, and Warren R. Wogen. $C^{\star}$-algebras generated by truncated Toeplitz operators. In Concrete operators, spectral theory, operators in harmonic analysis and approximation, volume 236 of Oper. Theory Adv. Appl., pages 181-192. Birkhäuser/Springer, Basel, 2014.

[10] Robert M. Gethner and Joel H. Shapiro. Universal vectors for operators on spaces of holomorphic functions. Proc. Amer. Math. Soc., 100(2):281-288, 1987.

[11] Gilles Godefroy and Joel H. Shapiro. Operators with dense, invariant, cyclic vector manifolds. J. Funct. Anal., 98(2):229-269, 1991.

[12] Sophie Grivaux. A new class of frequently hypercyclic operators. Indiana Univ. Math. J., 60(4):1177-1201, 2011.

[13] B. A. Lotto and D. Sarason. Multiplicative structure of de Branges's spaces. Rev. Mat. Iberoamericana, 7(2):183-220, 1991.

[14] Donald Sarason. Sub-Hardy Hilbert spaces in the unit disk, volume 10 of University of Arkansas Lecture Notes in the Mathematical Sciences. John Wiley \& Sons, Inc., New York, 1994. A Wiley-Interscience Publication.

[15] Donald Sarason. Algebraic properties of truncated Toeplitz operators. Oper. Matrices, 1(4):491-526, 2007.

[16] S. Shkarin. Orbits of coanalytic toeplitz operators and weak hypercyclicity. Submitted on arXiv:1210.3191.

[17] Stanislav Shkarin. Remarks on common hypercyclic vectors. J. Funct. Anal., 258(1):132-160, 2010.

[18] Daniel Suárez. Backward shift invariant spaces in $H^{2}$. Indiana Univ. Math. J., 46(2):593-619, 1997. 